\title{
Dense Hierarchy Decomposition for Bipartite Graphs
}

\author{
Edré Moreira, Guilherme Oliveira Campos, Wagner Meira Jr. \\ Universidade Federal de Minas Gerais, Brasil \\ \{edre, gocampos, meira\}@dcc.ufmg.br
}

\begin{abstract}
Dense subgraphs detection is a well known problem in Computer Science. Hierarchical organization of graphs as dense subgraphs, however, goes beyond simple clustering as it allows the analysis of the network at different scales. Despite the fact there are several works on hierarchical decomposition for unipartite graphs, only a few works for the bipartite case have been proposed. In this work we explore the problem of hierarchical decomposition of bipartite graphs. We propose an algorithm which we call weighted linking that produces denser and more compact hierarchies. The proposed algorithm is evaluated experimentally using several datasets and provided gains on most of them.
\end{abstract}

Categories and Subject Descriptors: H.2.8 [Database Management]: Database Applications

Keywords: bipartite graphs, dense subgraphs, graph mining, hierarchical decomposition

\section{INTRODUÇÃO}

Detecção de subgrafos densos é um assunto bem estudado em Ciência da Computação. A organização de um grafo como uma hierarquia de subgrafos densos, entretanto, vai além de agrupamentos simples, uma vez que permite a análise da rede em várias escalas. Decomposição hierárquica de grafos é aplicada em diversos cenários, como visualização de dados [Abello and Korn 2002], [Alvarezhamelin et al. 2006], detecção de anomalias [Shin et al. 2016], descoberta de subgrafos densos [Andersen and Chellapilla 2009], detecção de comunidades [Giatsidis et al. 2011], identificação de nós influentes [Kitsak et al. 2010], dentre outras. Considere, por exemplo, um grafo bipartido autorpublicação. Nos níveis mais profundos da hierarquia estão os autores que trabalham de forma mais integrada, representando grupos de pesquisa. Ao movermos para os níveis anteriores, encontramos autores que fazem pontes entre grupos ou colaboram de forma menos efetiva, e assim sucessivamente.

Formalmente, dado um grafo não ponderado e não direcionado $H=(N, L)$ e um subgrafo $H^{\prime}=\left(N^{\prime}, L^{\prime}\right)$, em que $H^{\prime} \subseteq H$, é comum encontrar na literatura as seguintes definições para densidade:

$$
\text { Densidade de grau médio: } d_{\text {grau }}=\frac{\left|L^{\prime}\right|}{\left|N^{\prime}\right|} \quad \text { Densidade de arestas: } d_{\text {arestas }}=\frac{\left|L^{\prime}\right|}{\left(\begin{array}{c}
\left|N^{\prime}\right| \\
2
\end{array}\right)} \text {. }
$$

Como um subgrafo trivial contendo dois vértices e uma aresta já possui a densidade máxima, considerando $d_{\text {arestas }}$, é é sabido que encontrar o máximo clique em um grafo é um problema NP [Tsourakakis et al. 2013], os autores trabalham com algoritmos aproximados para esse caso. Considerando $d_{\text {grau }}$, entretanto, existe solução polinomial para o problema [Goldberg 1984] .

Apesar de existirem vários trabalhos voltados para a decomposição hierárquica de grafos unipartidos, encontramos apenas um trabalho na literatura que trata do problema no caso de grafos bipartidos, considerando $d_{\text {arestas }}$ [Sariyüce and Pinar 2018]. Detectamos experimentalmente que o algoritmo proposto tende a produzir hierarquias com número de níveis excessivos, o que dificulta a interpretação. Além disso, subgrafos menos densos são gerados nos níveis intermediários, como discutiremos na Seção 4.

Copyright@2019 Permission to copy without fee all or part of the material printed in KDMiLe is granted provided that the copies are not made or distributed for commercial advantage, and that notice is given that copying is by permission of the Sociedade Brasileira de Computação. 
Tomemos, como exemplo, uma decomposição hierárquica de um grafo bipartido completo $K=$ $(U \cup V, E),|U|=m,|V|=n$ em $m$ subgrafos, onde o subgrafo mais externo contém todos os nós de $U$ e todos de $V$, o subgrafo no nível seguinte contém $m-1$ nós da partição $U$ e todos da partição $V$, e assim sucessivamente. Como todos os subgrafos possuem $d_{\text {aresta }}=1$, a densidade total será grande. Entretanto, a hierarquia não agrega informação, já que um único nível seria mais intuitivo. Assim, nosso objetivo é determinarmos uma decomposição que produza subgrafos mais densos e, ao mesmo tempo, com menos níveis hierárquicos.

Neste trabalho nós abordamos o problema da decomposição de um grafo bipartido estático, não ponderado e não direcionado, como uma hierarquia de subgrafos densos. Trabalhos futuros pretendem generalizar a solução.

As contribuições pretendidas por este trabalho podem ser resumidas a seguir:

-Algoritmo para decomposição hierárquica de grafos bipartidos: propomos o algoritmo weighted linking para decomposição hierárquica de grafos bipartidos que permite a definição do compromisso desejado entre altura da árvore e densidade dos subgrafos.

-Experimentos em bases de dados reais: apresentamos os resultados obtidos pelo algoritmo em bases de dados reais.

-Análise quantitativa: comparamos a nossa proposta com o algoritmo que representa o estado da arte, mostrando que podemos produzir melhores resultados.

-Análise qualitativa: como parte dos experimentos, realizamos uma análise qualitativa dos resultados em alguns cenários relevantes.

\section{TRABALHOS RELACIONADOS}

\subsection{Core decomposition}

Um $k$-core de um grafo G é o subgrafo maximal $H=\left(V_{H}, E_{H}\right) \mid \forall v \in V_{H}: \operatorname{deg} g_{H}(v) \geq k$, onde $\operatorname{deg}_{H}(v)$ é o grau do nó $v$ em $H$. O core number de um nó é o maior valor $k$ de um $k$-core contendo $v$. Em [Seidman 1983], o autor apresenta um algoritmo que computa o core number dos nós de um grafo em $O(n)$, sendo $n$ é o número total de nós.

Core decomposition é estendido para outros cenários além de grafos unipartidos, tais como grafos incertos [Bonchi et al. 2014], temporais [Wu et al. 2015], direcionados [Giatsidis et al. 2013], bipartidos [Batagelj and Zaversnik 2002], fluxos de grafos [Sarıüce et al. 2016], dentre outros.

\subsection{Detecção de subgrafos densos em grafos estáticos}

Existem diversos trabalhos abordando o problema de encontrar subgrafos densos considerando a densidade como o grau médio dos nós no subgrafo.

Em [Goldberg 1984], o algoritmo proposto computa, em tempo polinomial, a solução exata para este problema através do cálculo de $O(\log n)$ min-cut. Charikar propõe uma estratégia gulosa em [Charikar 2000] capaz de aproximar o subgrafo mais denso em $O(n)$, sendo $n$ o número de nós. Em [Khuller and Saha 2009], os autores estendem o algoritmo min-cut para encontrar subgrafos densos em grafos direcionados. Eles também propõem uma estratégia gulosa similar àquela proposta em [Charikar 2000] para grafos não direcionados. Assim como em [Charikar 2000], os autores provam que o algoritmo garante uma 2-aproximação para o problema de subgrafo mais denso em grafos direcionados.

Em [Tsourakakis et al. 2013], os autores relatam que a utilização de grau médio para detecção de subgrafos densos produz subgrafos grandes e com baixa densidade de arestas. Os autores definem uma função objetiva para encontrar a melhor $\alpha$-quasi-clique, ou seja, o melhor conjunto de vértices que 
maximiza a função. Os autores apresentam um algoritmo guloso, como uma pequena modificação naquele proposto em [Asahiro et al. 2000], e outro com uma heurística para busca local, que é provado ser localmente ótimo, ou seja, se qualquer vértice for adicionado ou removido do subgrafo, o valor da função objetiva é diminuído.

Em [Sariyüce and Pinar 2018], os autores propõem dois métodos para descobrir subgrafos densos, bem como para construir uma hierarquia, para o caso bipartido, denominadas tip decomposition e wing decomposition. Em tip decomposition, primeiramente é computado o número de borboletas que conecta cada dois nós na partição $U$. A princípio, para cada $u \in U$, o número de borboletas que $u$ participa, $\beta(u)$, é computado. Em seguida, para cada $u$ com o menor $\beta(u)$, o tip number $\theta(u)$ é atribuído como $\beta(u)$ e $\beta(v)$ é decrementado pelo número de borboletas compartilhadas entre $u$ e $v$, para cada nó $v$ envolvido em borboletas contendo $u$ e para os quais $\theta(v)$ não tenha sido computado, limitados por $\theta(u)$. O processo é similar para o wing decomposition. Entretanto, ao invés de serem computadas as borboletas para os nós, estas são computadas para as arestas. Em ambos os casos, a hierarquia de subgrafos é construída usando a estrutura de dados de conjuntos disjuntos [Sariyüce and Pinar 2016].

No trabalho [Sariyüce and Pinar 2016], os autores propõem um algoritmo para organizar subgrafos densos de forma hierárquica processando o grafo apenas uma vez. Pelo uso da estrutura de dados de floresta de conjuntos disjuntos, os subgrafos e a relação pai-filho é definida ao mesmo tempo que o core decomposition é executado. Ao final do processo de decomposição, um passo extra é feito para construir a hierarquia.

O trabalho apresentado aqui é uma extensão de [Sariyüce and Pinar 2016], dado que foi o único que encontramos aplicável ao problema proposto.

\section{DEFINIÇÃO DO PROBLEMA}

Consideremos o grafo bipartido $G=(U, V, E)$, onde $U$ e $V$ são conjuntos de nós, $U \cap V=\emptyset$, e $\{E \mid \forall e \in E: e=(u, v) \wedge u \in U \wedge v \in V\}$ é o conjunto de arestas, $H=\left(U_{H}, V_{H}, E_{H}\right) \subseteq G$.

Uma decomposição hierárquica $h$ de $G$ é composta por $k$ subgrafos aninhados $H_{1 . . k}$, com a única restrição de que $H_{i} \subset H_{j} \vee H_{j} \subset H_{i} \vee H_{i} \cap H_{j}=\emptyset \forall i, j \in 1 . . k$. O nível do subgrafo $H_{i}$ na decomposição hierárquica, sendo $H_{1}=G$, é definido como

$n\left(H_{i}\right)=\left\{\begin{array}{l}1, \text { se } i=1 \\ n\left(H_{i-1}\right)+1, \text { se } H_{i} \subset H_{i-1} \wedge \nexists H_{t} \mid H_{i} \subset H_{t} \subset H_{i-1} \forall t \in 1 . . k\end{array}\right.$

Definimos a altura total da hierarquia $h$ como $A_{h}=\sum_{i=1}^{k} n\left(H_{i}\right)$ e a densidade de arestas do subgrafo $H_{i}$ como $d\left(H_{i}\right)=\frac{\left|E_{H_{i}}\right|}{\left|U_{H_{i}}\right| x\left|V_{H_{i}}\right|}$. A densidade total da hierarquia $h$ é calculada pela equação $D_{h}=\sum_{i=1}^{k} d\left(H_{i}\right)$.

O problema tratado neste trabalho é encontrar a decomposição hierárquica $h$ de $G$ tal que a altura total, $A_{h}$, seja minimizada, ao mesmo tempo em que a densidade total, $D_{h}$, seja maximizada. Vale ressaltar que a decomposição hierárquica difere do agrupamento hierárquico visto que a união dos subgrafos localizados nas folhas da árvore hierárquica não necessariamente recompõem $G$, como ocorre naquele caso.

\section{HIERARQUIA DE SUBGRAFOS DENSOS POR WEIGHTED LINKING}

Tip decomposition e wing decomposition fazem a decomposição com base no número de borboletas (2,2-biclique) compartilhadas pelos nós, no primeiro caso, ou arestas, no segundo caso. Ambos os métodos aplicam a heurística apresentada em [Sariyüce and Pinar 2016] para construir os subgrafos e a hierarquia. Apesar da heurística ser capaz de construir a estrutura hierárquica, ela não considera o 


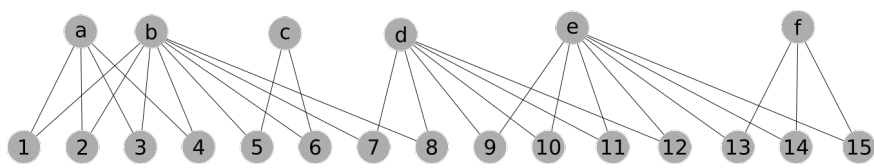

(a) Grafo autor-publicação

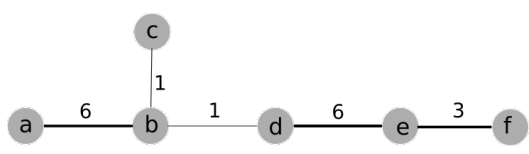

(b) Projeção em grafo unipartido

Fig. 1. Grafo autor-publicação e sua projeção em um grafo unipartido considerando borboletas compartilhadas.

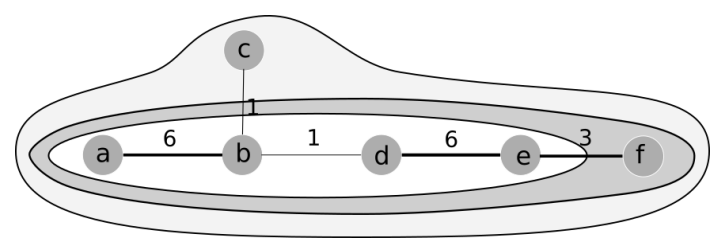

Fig. 2. Decomposição hierárquica por tip decomposition no grafo unipartido.

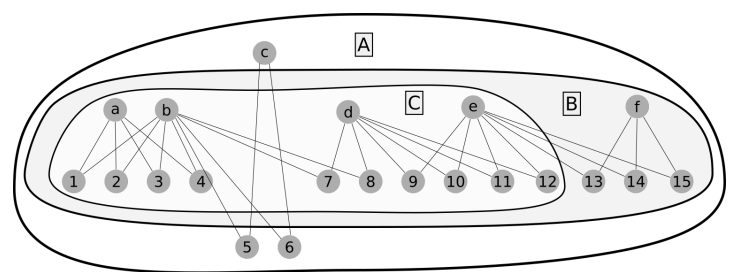

(a) Decomposição por tip decomposition

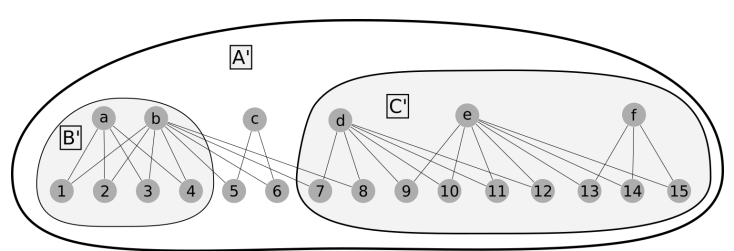

(b) Decomposição por weighted linking

Fig. 3. Decomposições do grafo da Fig. 1a.

peso das arestas entre subgrafos densos. Desta forma, subgrafos menos densos podem ser mesclados e gerar um subgrafo de densidade menor.

Podemos pensar no tip decomposition como uma decomposição da projeção do grafo bipartido. A projeção é construída como um grafo unipartido ponderado, tomando os nós de uma das partições e inserindo arestas conectando nós que compartilham borboletas; os pesos das arestas indicam o número de borboletas compartilhados por eles (Fig. 1b) ${ }^{1}$. O grau ponderado de cada nó é dado pela soma dos pesos das arestas incidentes. O processo de decomposição retira do grafo unipartido o nó de menor grau ponderado, atribuindo a seu tip number o valor de seu grau ponderado corrente. Ao remover o nó, o grau ponderado de seus vizinhos é ajustado, descontando o peso das arestas incidentes removidas. Esse processo continua até que todos os nós sejam processados. Nós conectados contendo o mesmo tip number, independente do peso da aresta que os conectam, são adicionados ao subgrafo, enquanto que nós conectados com tip number menores, independente do peso das arestas que os conectam, são posicionados em um nível mais acima na hierarquia (Fig. 2).

Consideremos o grafo autor-publicação da Fig. 1a, onde $\{a, b, c, d, e, f\}$ são autores e $\{1,2,3,4,5$, $6,7,8,9,10,11,12,13,14,15\}$ são publicações e sua projeção em um grafo unipartido ponderado da Fig. 1b. O peso das arestas, apresentado na projeção, indica o número de borboletas compartilhadas entre dois autores.

Ao executarmos o algoritmo tip decomposition, temos como resultado a hierarquia apresentada na Fig. 2 no grafo unipartido e, voltando ao grafo original, a hierarquia da Fig. 3a.

\footnotetext{
${ }^{1}$ Como aqui objetivamos construir uma hierárquica para os nós do grafo, consideraremos apenas o algoritmo tip decomposition para a análise. 


\begin{tabular}{c|c|c|c|c} 
Grafo & Nós na partição 1 & Nós na partição 2 & Total de arestas & Densidade de arestas \\
\hline Condmat & 16726 & 22015 & 58595 & 0,0001591292 \\
Marvel & 6486 & 12942 & 96662 & 0,001151536 \\
DBLP & 95580 & 93700 & 290365 & 0,00003242184 \\
Github & 56519 & 120867 & 440237 & 0,00006444427
\end{tabular}

Tabela I. Características das bases de dados.

Podemos observar que os níveis dos subgrafos na hierarquia são 1 , para o subgrafo $A ; 2$, para o subgrafo $B$; e 3, para o subgrafo $C$. As densidades de arestas $d\left(\right.$.) dos subgrafos são: $d(A)=\frac{30}{6 * 15}=0,33$, $d(B)=\frac{28}{5 * 13}=0,43$ e $d(C)=\frac{22}{4 * 10}=0,55$. Logo, a altura total é 6 e a densidade total é 1,31 .

Existem, entretanto, dois subgrafos densos bem definidos, que foram mesclados, induzidos por $a, b$ e por $d, e$, além de um nível extra gerado por $f$, que também está fortemente ligado a $e$.

Para contornar o problema apresentado, propomos o algoritmo weighted linking, que considera o tip number de cada nó e o peso das arestas para decidir pela adição ou não de um nó ao subgrafo. Pelo weighted linking, dois nós $u$ e $v$, unidos por uma aresta de peso $p$, devem ser mesclados se a seguinte condição for satisfeita, sendo $\alpha$ um parâmetro que regula o peso mínimo para a mesclagem:

$$
p>\alpha \frac{\theta(u)+\theta(v)}{2}
$$

Observemos a hierarquia da Fig. 3b, construída pelo algoritmo weighted linking com $\alpha=0,5$. Assim, os nós $a, b$ e $d, e$, que são unidos por uma aresta de peso abaixo do limiar, são mantidos em subgrafos distintos. Já o nó $f$ é adicionado ao subgrafo induzido por $d, e$, dado que está relacionado a este último por arestas de peso acima do limiar (Fig. 2).

Para a decomposição da Fig. 3b, os níveis dos subgrafos $A^{\prime}, B^{\prime}$ e $C^{\prime}$ na hierarquia são, respectivamente, 1,2 e 2 . Já as densidades de arestas são as seguintes: $d\left(A^{\prime}\right)=\frac{30}{6 * 15}=0,33, d\left(B^{\prime}\right)=\frac{8}{2 * 4}=1 \mathrm{e}$ $d\left(C^{\prime}\right)=\frac{16}{3 * 9}=1,93$.

Assim, a altura total é 5, e a densidade total é 1,93. Temos, pois, uma decomposição que possui uma hierarquia menos profunda e com uma densidade total maior do que aquela da Fig. 3.

\section{RESULTADOS EXPERIMENTAIS}

\subsection{Bases de dados}

Nós avaliamos o weighted linking nas mesmas bases de dados utilizadas em [Sariyüce and Pinar 2018]. Algumas bases foram suprimidas por restrição de espaço. DBLP também foi reduzida por restrições de hardware.

DBLP$^{2}$ é um grande repositório bibliográfico de ciência da computação, contendo metadados de publicações das principais conferências e periódicos da área. O grafo bipartido autor-publicação foi construído utilizando um subconjunto de dados contendo publicações de 23 conferências, a saber: AAAI, IAAI, CIKM, CVPR, ECIR, ECML, PKDD, EDBT, ICDT, ICDE, ICDM, ICML, IJCAI, KDD, PAKDD, PODS, SDM, SIGIR, SIGMOD, SSDBM, VLDB, WSDM, e WWW. Condmat ${ }^{3}$ [Kunegis 2013] é um grafo bipartido que modela relacionamentos entre autores e publicações na seção Condensed Matter do sítio $a r \mathrm{Xiv}^{4}$. Uma partição representa autores, enquanto que a outra representa publicações. Uma aresta entre as partições indica que um autor participou daquela publicação. Github ${ }^{5}$ [Kunegis

\footnotetext{
${ }^{2}$ http://dblp.uni-trier.de/db/

${ }^{3}$ http://konect.uni-koblenz.de/networks/opsahl-collaboration

${ }^{4}$ https://arxiv.org/

${ }^{5}$ http://konect.uni-koblenz.de/networks/github
} 


\begin{tabular}{c|c|c|c} 
Grafo & $\alpha$ & Incremento na densidade total & Decremento na altura total \\
\hline Condmat & 0,4 & $3,97 \%$ & $11,53 \%$ \\
Marvel & 0,15 & $7,13 \%$ & $1,8 \%$ \\
DBLP & 0,4 & $5,07 \%$ & $6,93 \%$ \\
Github & 0,15 & $-1,15 \%$ & $-10,60 \%$
\end{tabular}

Tabela II. Ganhos obtidos pela execução de weighted linking com o melhor parâmetro $\alpha$

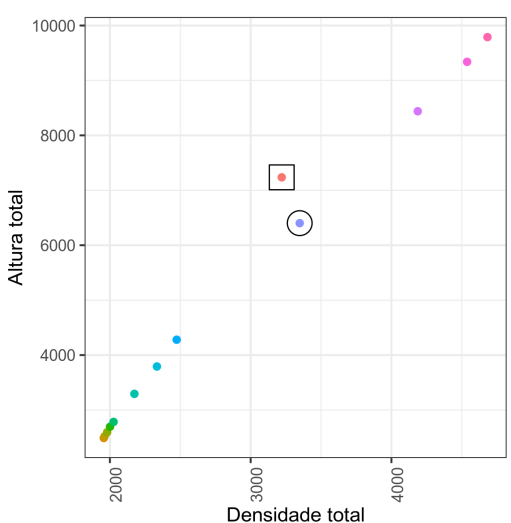

(a) Condmat

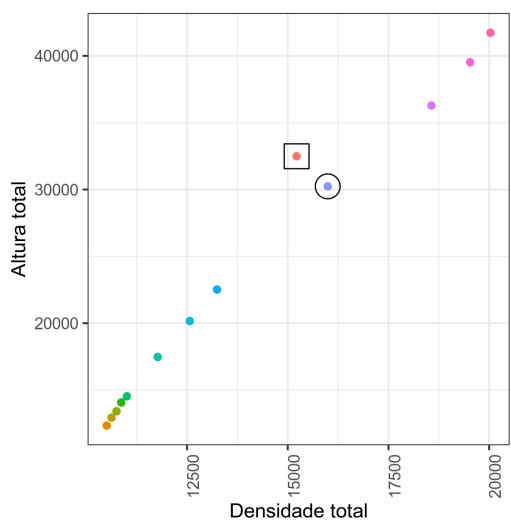

(c) $D B L P$

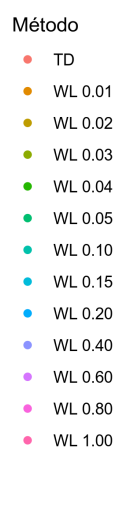

Método

- TD

- WL 0.01

- WL 0.02

- WL 0.03

- WL 0.04

- WL 0.05

- WL 0.10

- WL 0.15

- WL 0.20

- WL 0.40

- WL 0.60

- WL 0.80

- WL 1.00

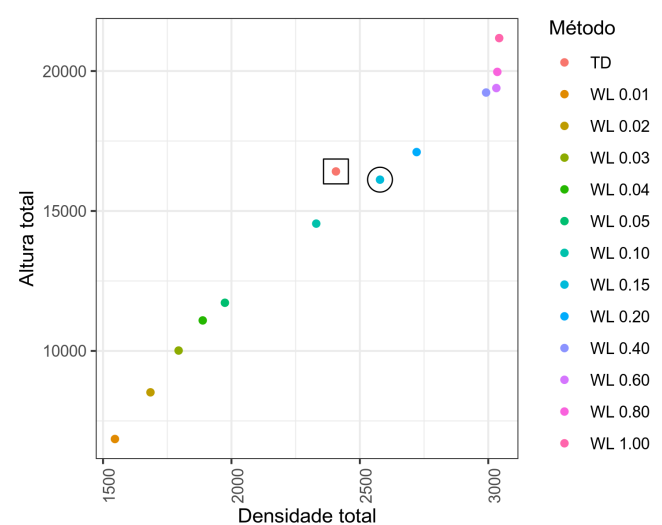

(b) Marvel

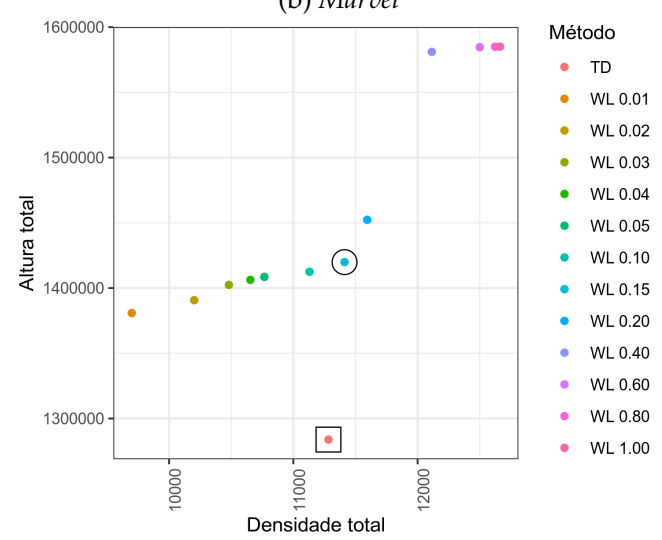

(d) Github

Fig. 4. Representação da altura total versus densidade total para a decomposição hierárquica dos grafos pelo algoritmo weighted linking (WL), variando o $\alpha$, e por tip decomposition (TD). O quadrado destaca o resultado para TD, enquanto que o círculo destaca um resultado para WL mais próximo de TD .

2013] modela a rede de associação entre desenvolvedores e projetos no sítio Github ${ }^{6}$. O grafo bipartido contém arestas indicando que um usuário é membro de um projeto. Marvel$^{7}$ [Alberich et al. 2002] modela a relação de ocorrência entre personagens Marvel e revistas em quadrinho.

A Tabela I apresenta algumas características das bases de dados utilizadas nos experimentos.

\subsection{Análise dos resultados}

De uma forma geral notamos, pela Fig. 4, que valores mais baixos para $\alpha$ tendem a gerar hierarquias menores e, por consequência, menos densas, enquanto que valores mais altos geram hierarquias

\footnotetext{
${ }^{6}$ https://github.com/

${ }^{7}$ http://bioinfo.uib.es/ joemiro/marvel/porgat.txt 


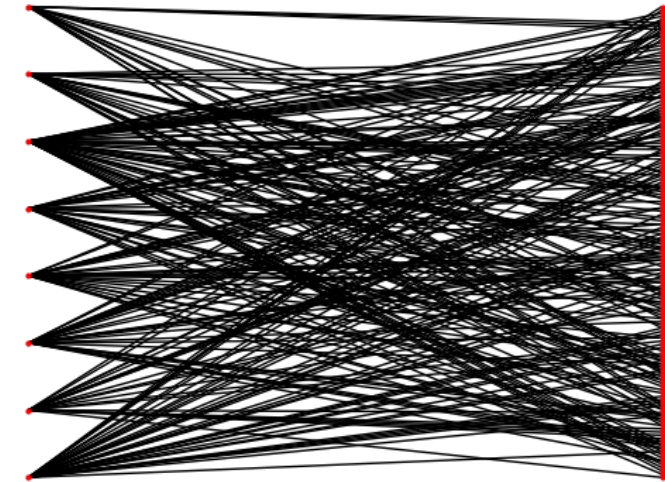

(a) Tip decomposition

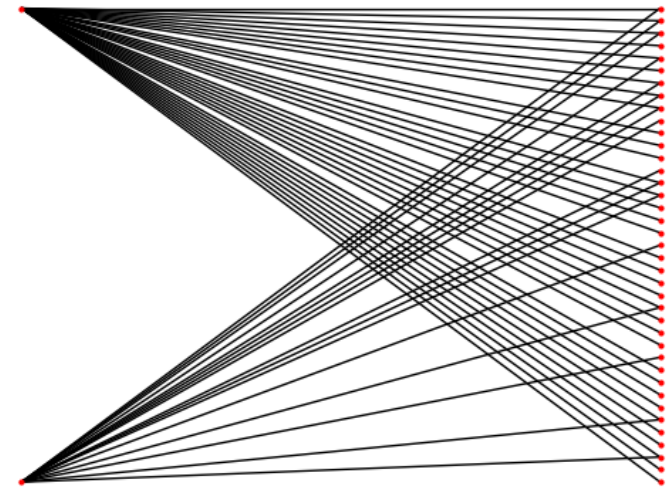

(b) Weighted linking

Fig. 5. Subgrafos extraídos da base de dados DBLP.

maiores e mais densas. A explicação para este fenômeno é que, com valores menores do parâmetro, o número de borboletas (ou peso da aresta no grafo unipartido) para promover a junção de nós em um mesmo subgrafo é menor, o que favorece essa situação. Os gráficos evidenciam, também, o compromisso entre o número de níveis da hierarquia e a densidade dos subgrafos.

A Fig. 4 mostra a decomposição hierárquica dos grafos Condmat, Marvel, DBLP e Github por weighted linking, com o parâmetro $\alpha$ assumindo os valores de 0,01 a 1, bem como por tip decomposition. Podemos observar que, para os três primeiros grafos, os pontos em destaque gerados por weighted linking, com $\alpha$ igual a $0,4,0,15$ e 0,4 , respectivamente, apresentam hierarquias menores e com densidades maiores que tip decomposition.

Para o grafo Github, entretanto, tip decomposition apresentou um melhor resultado. A pequena variação da altura total com a variação de $\alpha$ de 0,01 a 0,2 promove separações pontuais em subgrafos que geram grande ganho em densidade. Os motivos para a diferença de qualidade entre os métodos, contudo, devem ser melhor analisados. A Tabela II apresenta um resumo dos ganhos ou perdas em cada caso .

A Fig. 5 apresenta dois subgrafos, mais internos na hierarquia, em que figura um determinado autor. O subgrafo gerado por tip decomposition (Fig. 5a) contém 8 autores, 146 publicações e 227 relacionamentos autor-publicação, apresentando uma densidade de 0,194. Aqui, a rede do autor em questão inclui co-autores de seus co-autores, que não compartilham publicações com ele. Já o subgrafo gerado por weighted linking (Fig. 5b) contém 2 autores, 39 publicações e 51 relacionamentos autorpublicação, com uma densidade total de 0,653 . Neste caso, apenas um dos co-autores, que possui número mais significativo de publicações em comum, foi incluído, eliminando as conexões indiretas. O grafo gerado por weighted decomposition é, assim, significativamente mais denso, evidenciando a efetividade do algoritmo na geração de subgrafos mais relevantes.

\section{CONCLUSÃO E TRABALHOS FUTUROS}

Neste trabalho tratamos o problema de decomposição hierárquica de grafos bipartidos em subgrafos densos. Propusemos o algoritmo weighted linking para equilibrar o compromisso entre o número de níveis hierárquicos e a densidade total da decomposição. Mostramos, experimentalmente, que o algoritmo é capaz de gerar hierarquias menores e mais densas do que o algoritmo que representa o estado da arte. Apresentamos, ainda, uma análise qualitativa no grafo DBLP. Evidenciamos, experimentalmente, que existe um compromisso entre o número de níveis hierárquicos e a densidade total da hierarquia.

Não encontramos na literatura, entretanto, métricas que possibilitem avaliação objetiva da quali- 
dade de decomposições hierárquicas de grafos, o que propomos como trabalhos futuros. Pretendemos conduzir experimentos com um número maior de bases de dados para validar a solução. Pretendemos, também, trabalhar na exploração do espaço paramétrico, de forma a tentar estimar o valor ideal do parâmetro $\alpha$ que produz o resultado mais próximo do ótimo.

\section{AGRADECIMENTOS}

Os autores agradecem à FAPEMIG, CNPq e CAPES pelo apoio financeiro. Este trabalho também foi parcialmente financiado pelos projetos InWeb, MASWeb, EUBra-BIGSEA, INCT-Cyber e ATMOSPHERE.

\section{REFERÊNCIAS}

Abello, J. And Korn, J. Mgv: A system for visualizing massive multidigraphs. IEEE Transactions on Visualization and Computer Graphics 8 (1): 21-38, 2002.

Alberich, R., Miro-Julia, J., And Rossello, F. Marvel universe looks almost like a real social network, 2002. cite arxiv:condmat/0202174Comment: 14 pages, 3 figures.

Alvarez-hamelin, J. I., Asta, L. D., Barrat, A., And Vespignani, A. Large scale networks fingerprinting and visualization using the k-core decomposition. In Advances in Neural Information Processing Systems 18, Y. Weiss, B. Schölkopf, and J. C. Platt (Eds.). MIT Press, pp. 41-50, 2006.

Andersen, R. and Chellapilla, K. Finding dense subgraphs with size bounds. In Algorithms and Models for the Web-Graph, K. Avrachenkov, D. Donato, and N. Litvak (Eds.). Springer Berlin Heidelberg, Berlin, Heidelberg, pp. 25-37, 2009.

Asahiro, Y., Iwama, K., Tamaki, H., And Tokuyama, T. Greedily finding a dense subgraph. Journal of Algorithms 34 (2): 203-221, 2000.

Batagelj, V. And ZaversniK, M. Generalized cores. CoRR vol. cs.DS/0202039, 2002.

Bonchi, F., Gullo, F., Kaltenbrunner, A., And Volkovich, Y. Core decomposition of uncertain graphs. In Proceedings of the 20th ACM SIGKDD International Conference on Knowledge Discovery and Data Mining. KDD '14. ACM, New York, NY, USA, pp. 1316-1325, 2014.

Charikar, M. Greedy approximation algorithms for finding dense components in a graph. In Proceedings of the Third International Workshop on Approximation Algorithms for Combinatorial Optimization. APPROX '00. Springer-Verlag, Berlin, Heidelberg, pp. 84-95, 2000.

Giatsidis, C., Thilikos, D. M., And Vazirgiannis, M. Evaluating cooperation in communities with the k-core structure. In 2011 International Conference on Advances in Social Networks Analysis and Mining. pp. 87-93, 2011.

Giatsidis, C., Thilikos, D. M., And Vazirgiannis, M. D-cores: measuring collaboration of directed graphs based on degeneracy. Knowledge and information systems 35 (2): 311-343, 2013.

Goldberg, A. V. Finding a maximum density subgraph. Tech. rep., Berkeley, CA, USA, 1984.

Khuller, S. AND SAHA, B. On finding dense subgraphs. In Proceedings of the 36th International Colloquium on Automata, Languages and Programming: Part I. ICALP'09. Springer-Verlag, Berlin, Heidelberg, pp. 597-608, 2009.

Kitsak, M., Gallos, L. K., Havlin, S., Liljeros, F., Muchnik, L., Stanley, H. E., and Makse, H. A. Identification of influential spreaders in complex networks. Nature physics 6 (11): 888, 2010.

Kunegis, J. Konect: The koblenz network collection. In Proceedings of the 22Nd International Conference on World Wide Web. WWW'13 Companion. ACM, New York, NY, USA, pp. 1343-1350, 2013.

SARIYÜCe, A. E., GediK, B., Jacques-Silva, G., Wu, K.-L., AND ÇATAlYüreK, Ü. V. Incremental k-core decomposition: algorithms and evaluation. The VLDB Journal-The International Journal on Very Large Data Bases 25 (3): 425-447, 2016.

SARIYÜCE, A. E. AND PINAR, A. Fast hierarchy construction for dense subgraphs. Proceedings of the VLDB Endowment 10 (3): 97-108, 2016.

SAriyüce, A. E. And PInAR, A. Peeling bipartite networks for dense subgraph discovery. In Proceedings of the Eleventh ACM International Conference on Web Search and Data Mining. WSDM '18. ACM, New York, NY, USA, pp. 504-512, 2018.

SeIdman, S. B. Network structure and minimum degree. Social networks 5 (3): 269-287, 1983.

Shin, K., Eliassi-Rad, T., AND Faloutsos, C. Corescope: Graph mining using k-core analysis - patterns, anomalies and algorithms. In 2016 IEEE 16th International Conference on Data Mining (ICDM). pp. 469-478, 2016.

Tsourakakis, C., Bonchi, F., Gionis, A., Gullo, F., And Tsiarli, M. Denser than the densest subgraph: Extracting optimal quasicliques with quality guarantees. In Proceedings of the 19th ACM SIGKDD International Conference on Knowledge Discovery and Data Mining. KDD '13. ACM, New York, NY, USA, pp. 104-112, 2013.

Wu, H., Cheng, J., Lu, Y., Ke, Y., HuAng, Y., YAN, D., AND Wu, H. Core decomposition in large temporal graphs. In 2015 IEEE International Conference on Big Data (Big Data). pp. 649-658, 2015. 